\title{
Spatio-Temporal Shape Parameterization of the Human Ventricles
}

\author{
Sándor M. Szilágyi \\ Department of Informatics, Petru Maior University \\ Str. Nicolae Iorga Nr. 1, 540088 Tîrgu Mureş, Romania \\ sandor.szilagyi@science.upm.ro
}

\begin{abstract}
This paper gives a solution for improving the geometric estimation of the human ventricles, by reducing their shape estimation error. The parametric description of the studied organ can be performed at arbitrary resolution during the whole visualization process. After the problem description, the paper presents each main step of the proposed shape estimation algorithm. The presented method determines not only the general shape of the ventricles, but the internal tissue direction, too. The estimation error decreases more than 10 times if the resolution is increased by 3 times. The biological parameters like gender or age also affect the estimation performance. The obtained accuracy of the method was slightly higher in the case of female hearts and for the left ventricle. Finally, concluding remarks emphasize some important features of the given approach and the future research directions.
\end{abstract}

Keywords: ventricle modeling; heart geometry; shape estimation; interpolation techniques

\section{Introduction}

Cardiac disease, despite the continuous progress of medical health care and intensive research, still represents the main mortality factor in many low- and middle-income countries. The main reason of slow progress represents the partially understood heart functioning [1].

Sudden cardiac death, mostly caused by ventricular fibrillation (VF), represents an important cause of mortality. In spite of this consideration, the electric activity and mechanical mechanisms responsible for VF are only partially discovered. It would be utmost important to understand how the onset of arrhythmias that cause fibrillation depends on details of the cardiac activity, such as the size of ventricles [2], geometry [3], mechanical [4] and electrical state [5], anisotropic fiber structure [6] and inhomogeneity [7].

A proper presentation of the cardiac activity involves the description of its electric and mechanic properties. The spread of electric excitation wave in the cardiac 
muscle determines the nature of contraction, but several important aspects, such as pumping strength and volume cannot be deduced without a detailed mechanical analysis of the heart [8].

One of the most important cardiac pumping factors is the structural architecture of the ventricles [9]. Albeit several general geometric factors such as shape and size of ventricles, wall thickness and structure play a critical role in medical diagnosis, the proper orientation of cardiac muscle fibers is crucial for an adequate contraction [10]. There are several cardiac affections and traumatic events such as cardiac infarction [11] and myocardial fibrosis [12] that may induce the misalignment of ventricular fibers, developing reduced cardiac pump activity that may lead to the rise of further cardiac disorders [13].

Taking the abovementioned considerations into account is imperative for a computational ventricular model that is able to predict accurately the electric and mechanic function of the heart for normal and pathological cases, to involve not only the general description parameters but the distribution of fiber orientations too.

There exist several methods to determine the shape and structure of the ventricles. The anatomy of the whole heart and the overall placement of the cardiac muscle fibers is described by Streeter and Hanna [14], while the orientation changes from epicardium to endocardium is presented by Arts et al [15] and Geerts et al [16]. From anatomical measurements it is concluded that the septal and left ventricle walls fiber orientations are similar.

In spite of wide agreement on fiber directions in ventricular tissue there is no generally accepted concept on how to assign these orientations to realistic computerized ventricular models. Göktepe and Kuhl have used a simplified representation, where the ventricles are considered nested and truncated ellipsoids [17]. Takayama proposed a geometrical-based approach to generate a layered ventricular structure [18].

A geometric approximation of the heart may introduce significant errors. A proper mapping of a non-ellipsoidal form to an idealized ellipsoidal one raises serious architectural deformations especially in pathological cases. Moreover, inherent singularities may appear in the approximation of the septal and apex region [19]. To solve these problems, Kotikanyadanam et al have used various 3D imaging techniques during the development of a patient-specific ventricular geometry model [20], where the fiber orientation in the patient-specific ventricular geometry was determined by a Poisson equation-based interpolation technique [8].

Biological systems represent the most complex studied architectures. Their proper description is possible via models that always represent only a simplification of the investigated object. The reductionist approach applied in the case of complex heart modeling demands a simplified description of the whole ventricle. The cell level geometric description of the ventricles is difficult due to the immense 
parameter number, obscure biological information and low computational power, necessitating the usage of biological model parameters.

Our goal in this paper is to present the development of a spatio-temporal ventricular geometry estimation method that involves the anatomical structure of the human ventricles. The rest of the paper is organized as follows: Section 2 gives a detailed description of the geometry estimation method, involving the effect of electric and mechanic properties of the ventricles, studied for normal and pathological cases. We outline the effect of the various parameter alterations on the generated activation potential (AP). Section 3 presents and discusses several aspects of the model functionality and the results of simulations carried out using the model. Finally, in Section 4, the conclusions are formulated.

\section{Materials and Methods}

\subsection{Motivation of Shape Parameterization}

The real-time visualization and analysis of the human heart, using up-to-date representation techniques is partially solved. The main problem is the inability of current imaging systems to yield both visual information and internally state parameters. Moreover, several imaging techniques are considered invasive.

The above mentioned problem can be partially solved using computational models that beside the visual representation may give the necessary state parameters. All computational model representation strategy uses an indirect way to obtain the investigated parameters. Physicians have no possibility to measure in real time all requested data, so they have to predict these values using computational models.

Many physiological deficiencies are related to the geometry of heart. These geometric alterations are reflected in the modified values of several medical parameters, thus obtained medical parameters are not always suitable for recent computerized heart model-based visualization techniques.

Computerized data visualization is based on voxels, pixels and triangles. The determined medical information has to be transformable to these basic geometryrelated computational data. By using a heart shape parameterization method, we want to realize a transformation of medical data into basic geometry elements via a set of engineer-related parameters. This process is visualized in Fig 1. The adaptive aspect of these inner-level parameters, independently from the used resolution of the geometric representation, allows a detailed description of the heart.

In Fig. 1 the dashed line from the anatomical knowledge box and visualization data element toward the intermediate parameters box represent the possibility of 
determination of several intermediate parameters. In addition, the calculated internal parameters may allow the estimation of several medical parameters. This complete loop may be used for modeling or system validation purposes.

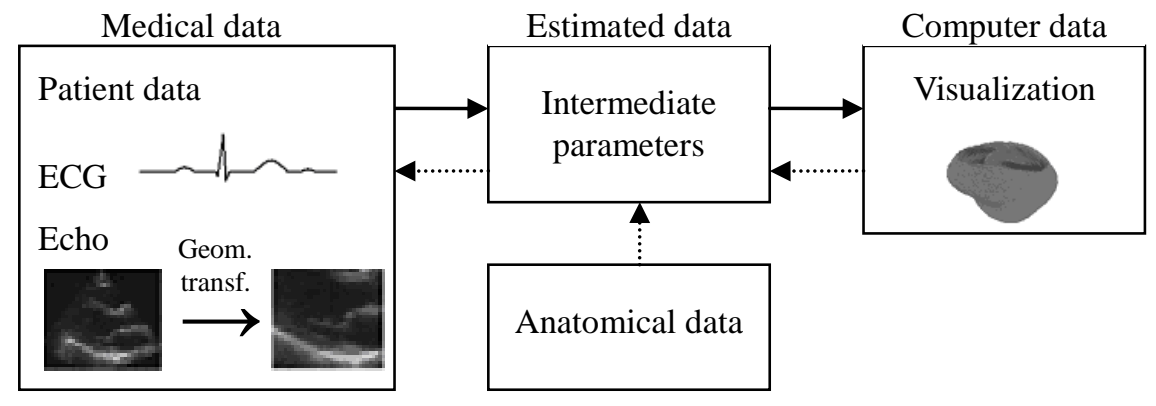

Figure 1

The medical data and graphical visualization data are connected to the estimated engineer-related parameters. The anatomical considerations and visualization data may be used to determine several medical parameters

A real time spatial heart visualization method uses an engineered parametric model, and is able to show all geometrical changes that take place during a whole normal or pathologic cardiac cycle. The lack of information about the effect of various clinical conditions, such as ischemia, on the heart functionality demands the development of intuitive modeling tools that are capable to handle multiple cardiac layers simultaneously. This modeling approach may enhance the detection rate of various phenomena that are related to the irregular contraction of the heart.

An important aspect of this parameter organization is the possibility to determine many internal parameters from raw computerized geometrical data. The set of voxels are organized in triangles, and the connection between them determines the inner and outer surface of the ventricles. Using our anatomical knowledge concerning the structure of human ventricles, allows the determination of many internal parameters.

\subsection{Methodology of Cardiac Data Processing}

The determination of the geometry of heart ventricles properly represents the solution many physiological problems. Normally, the left ventricle can be estimated by an ellipsoidal shaped object, but neither the wall thickness nor tissue orientation is homogenous. Moreover, the possible alterations from the normal shape due several pathological injuries may dramatically increase the estimation errors. Several papers describe different approximation methods [8], but none of the proposed methods are suitable for all pathological cases. 
In our approximation, proper heart geometry estimation is based on the followings:

- Anatomical data of the ventricles, obtained from medical data libraries

- Shape estimation based on direct measurements (for example echocardiography measurements)

- Introduction of several auxiliary medical data such as age, gender, position, ECG signal, blood pressure and respiration

The combination of these data may enhance the precision of approximation of the ventricles boundaries. In 1991 Nielsen et al. [21] have determined the fiber orientation in ventricular tissue. Based on their study it is assumed that all fibers in the epicardium and right endocardium (considered the epicardium of the left ventricle) are inclined forward at $70^{\circ}$, and the endocardial fibers of the left and right ventricles (except the septal region) are inclined backward at $80^{\circ}$. The inclination of the middle regions takes intermediate values, depending on their position.

The key idea in the applied fiber orientation determination algorithm is to generate a smooth coordinate-free interpolation of the spatial directions of the fibers involved in all clusters [22, 23]. The intermediate ventricular tissue layers do not have a precisely determined structure, so all internal compartments should be described by a guiding parameter that determines how the studied compartment behaves related to the endo- and epi-cardial tissue. We assume that a given compartment has a $p_{\text {endo }}, p_{\text {epi }}$ and $p_{\text {middle }}$ probabilities to belong to the endocardium, epicardium or intermediate region, so the spatial direction $d$ of the compartments tissue is:

$$
d_{\text {comp. }}(x, y, z)=p_{\text {endo }}\left(f_{r}, x, y, z\right)+p_{\text {epi }}\left(f_{r}, x, y, z\right)+p_{\text {middle }}\left(f_{r}, x, y, z\right) \text {, }
$$

where $f$ represents the distance rate of the compartment from the base plane related to the whole base-apex distance, the index $r$ defines the region of the compartment (for example left or right ventricle) and the symbols $x, y, z$ show the three perpendicular spatial directions.

Since the visual interpretation of the heart using ultrasound (US) representation techniques cannot yield as accurate data as CT and MRI images, the ventricular wall detection procedure demands a deeper usage of the a priori information. The sensibility of US imaging to noise and reflections implies an intelligent filtering. In spite of these problems, a large number of images may yield adequate results, but it is necessary to use an automatic border detection (ABD) algorithm that contains the following steps [24]:

- Pre-processing (smoothing, contrast checking)

- Edge or region detection (thresholds, edge detectors) 
- Geometric object models (for example radial search)

- Anatomical structure model

- Interpretation (based on high-level knowledge)

The enrolled disadvantages convinced us to use an Active Appearance Model (AAM) that was presented in [24]. Its main steps are:

- Extract the average organ shape

- Extract the principal shape variations

- Create the appearance model

- Generate probable echocardiography image

- Find the desired structure by error minimization technique

\subsection{Estimation of Geometrical Data}

The estimation of geometric data is a multi-level process. The ventricular tissue has a multi-layered structure, so we have to estimate the barrier of each layer. Most geometric determination methods define the inner and outer surface of the ventricles.

All determined surfaces are constituted by a list of description points (DP) described by spatial coordinates, so the list $L$ can be presented as:

$L_{\text {Surf. }}=\left\{P_{1}\left(x_{1}, y_{1}, z_{1}\right), \ldots, P_{k}\left(x_{k}, y_{k}, z_{k}\right)\right\}$,

where $k$ represents the number of points in the list and the symbols $x, y, z$ show the three perpendicular spatial directions. We have to use separate lists for the inner and outer surfaces, however they may describe the surface of a large object, for example the outer surface of the whole heart. It is important to mention that the lists can be partially combined, so from the list of outer surfaces of the whole heart, we can only partially deduce the outer surface of the left ventricle.

In the classical approach, the resolution of the simulation highly depends on the number of points from the surface list. In order to create a high-resolution simulation from a relatively low number of DPs, we have to interpolate the surface of the studied organ. The interpolation is performed for both the inner and outer surfaces.

To accomplish the interpolation it is necessary to execute the following data processing steps iteratively:

- Triangulation

- Division of the junction lines

- Estimation of the spatial coordinates on the divided lines 
We used the Delaunay triangulation method [25], that can generate a set of triangles, in such a way, that $P$ is not defined beforehand. It is supposed, that the points in the surface list are not in a line. The Delaunay triangulation method maximizes the minimum angle of all generated triangles, so it tends to avoid narrow triangles.

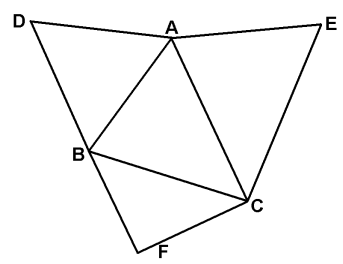

(A)

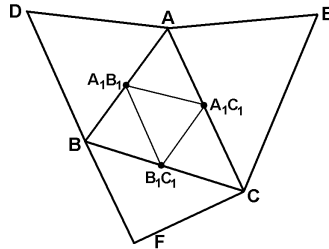

(B)

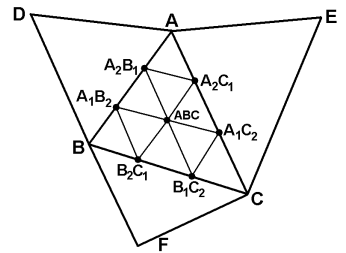

(C)

Figure 2

Generation of additional spatial triangles on the surface of the modeled object applying various resolutions. For image (A) $d f=1$, for image (B) $d f=2$ and for image (C) $d f=3$

The next step represents the generation of a high resolution triangulated surface of the spatial object. Now the goal is the construction of a surface generated by $q$ DP$s$, where $q>>p$. In the following, we will determine the proper division factor $(d f)$. It is important to mention that usually $d f$ will not be an integer value, so the processing method must handle these cases.

Figure 2 presents the decomposition of the inner triangle into sub-triangles (A). The sub-figure (B) present the decomposition for $d f=2$, while sub-figure (C) shows the same processing for $d f=3$. It can be observed that for a given positive integer $d f$, on the edges of the inner triangle there will appear $E P=3 \cdot(d f-1)=3 \cdot d f-3$ external points $(E P)$. The number of internal points $(I P)$ in the inner triangle for the same integer $d f$ will be given by:

$$
I P=\left[(d f-2)^{2}+(d f-2)\right] / 2=\left[d f^{2}-3 \cdot d f+2\right] / 2 .
$$

Each EP is a member point of two triangles, so the necessary points to perform at integer $d f$ will be approximated as:

$$
\begin{aligned}
q & =p+t \cdot(E P / 2+I P) \approx p \cdot\left(\frac{3 \cdot d f-3+d f^{2}-3 \cdot d f+2+2}{2}\right), \\
& =p \cdot \frac{d f^{2}+1}{2} \approx p \cdot \frac{d f^{2}}{2}
\end{aligned}
$$

where $t$ represent the number of triangles. 
During the reverse problem, the necessary $d f$ in presence of $p$ DP-s and $q$ aimed DP's will be $d f \approx \sqrt{2 q / p}$. It is observable that the relation yields proper results for $q>>p$.

For a large, closed-surfaced normal spatial object, where $p$ DP-s exist that form $t$ triangles, it can be considered that the number of triangles and points are approximately the same, so $p \approx t$. In this case the total number of side-edges (se) will be $s e=p+t-1 \approx 2 \cdot p$.

In the likely case of non-integer $d f$, the division of some triangles may be performed partially. This partial division may create ambiguous results, where the selected solution possesses the more balanced angles and sizes. The fractional division of a triangle is presented in Figure 3.

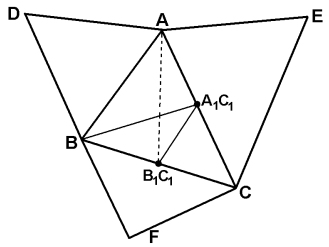

(A)

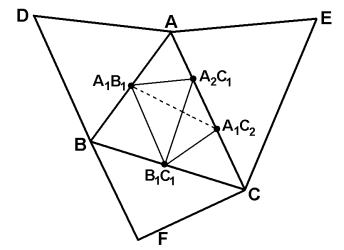

(B)

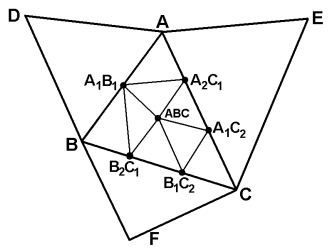

(C)

Figure 3

Several cases of fractional division factor. Image (A) and (B) present a simple ambiguous case, while (C) present a unique solution

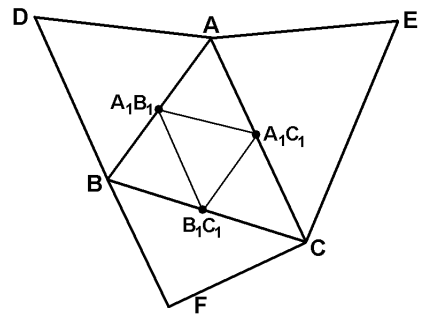

(A)

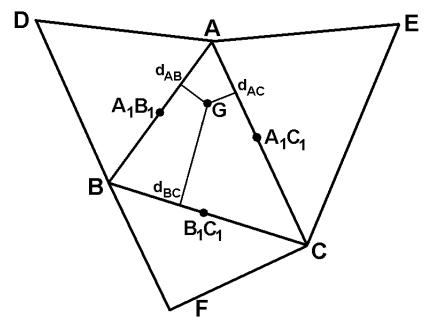

(B)

Figure 4

Determination of spatial coordinates of the internal points. Image (A) shows the case of coplanar $A, B$,

$C$ and $F$ points. Image (B) visualizes the distances of the point $\mathrm{G}$ from the three side-edges

It is important to reduce the number of narrow triangles, so the division is performed more often on the longer side-edges of the triangles. In case of multiple solutions we selected the shorter diagonal line of the trapezoids. 
The estimation of spatial coordinates of each created division point is realized by a spherical interpolation method, based on the fact that every non-coplanar four points are situated at the surface of a sphere.

The situation of coplanar points is quite simple. Suppose that points $A, B, C$ and $F$ from Figure 4(A) are coplanar. In this case we can affirm that all points of segment $B C$ are in the same plane, and the centre of the circumscribed sphere is in the infinite.

If the $A, B, C$ and $D$ points are non-coplanar, the centre of the circumscribed sphere obeys the rule: $d_{O A}=d_{O B}=d_{O C}=d_{O D}$, so the spatial coordinates of the unique $O$ can be determined by solving the equation:

$$
\left|\begin{array}{ccccc}
x^{2}+y^{2}+z^{2} & x & y & z & 1 \\
x_{A}^{2}+y_{A}^{2}+z_{A}^{2} & x_{A} & y_{A} & z_{A} & 1 \\
x_{B}^{2}+y_{B}^{2}+z_{B}^{2} & x_{B} & y_{B} & z_{B} & 1 \\
x_{C}^{2}+y_{C}^{2}+z_{C}^{2} & x_{C} & y_{C} & z_{C} & 1 \\
x_{D}^{2}+y_{D}^{2}+z_{D}^{2} & x_{D} & y_{D} & z_{D} & 1
\end{array}\right|=0 .
$$

Once the spatial centers of spheres $S_{A B C D}, S_{A B C E}, S_{A B C F}$ are determined, it is possible to determine the sphere centre that contains point $G$. As presented in Figure 4(B), the $X$ coordinate of sphere centre on whose surface point $G$ is satiated will be:

$S_{G_{X}}=\left(\frac{1}{d_{A B}}+\frac{1}{d_{A C}}+\frac{1}{d_{B C}}\right)^{-1}\left(\frac{S_{A B C D_{X}}}{d_{A B}}+\frac{S_{A B C E_{X}}}{d_{A C}}+\frac{S_{A B C F_{X}}}{d_{B C}}\right)$.

The $Y$ and $Z$ coordinates of the sphere center may be determined in an analogous way.

\section{Results}

The level of geometry estimation error depends on the granularity of representation and the shape of modeled object (see Figures 5 and 6). In our case, the reference constitutes a high-resolution double layered triangle mesh object that involves both ventricles, containing 20000 triangles. For interpolation purposes, a subset of them was selected that must contain more than 1800 triangles. The distances of the compartments were determined from the middle of the AV-node. Figure 5 presents the simulated geometric estimation error plotted against the division factor. The applied granularity has a linear scale, while the estimation error is presented in logarithmic range. 
Figure 6 indicates the geometric estimation error for a healthy male (M) and female $(F)$ heart. The solid line represents the left, while the dashed line shows the right ventricle estimation error plotted against the distance from the AV-node (base of ventricles).

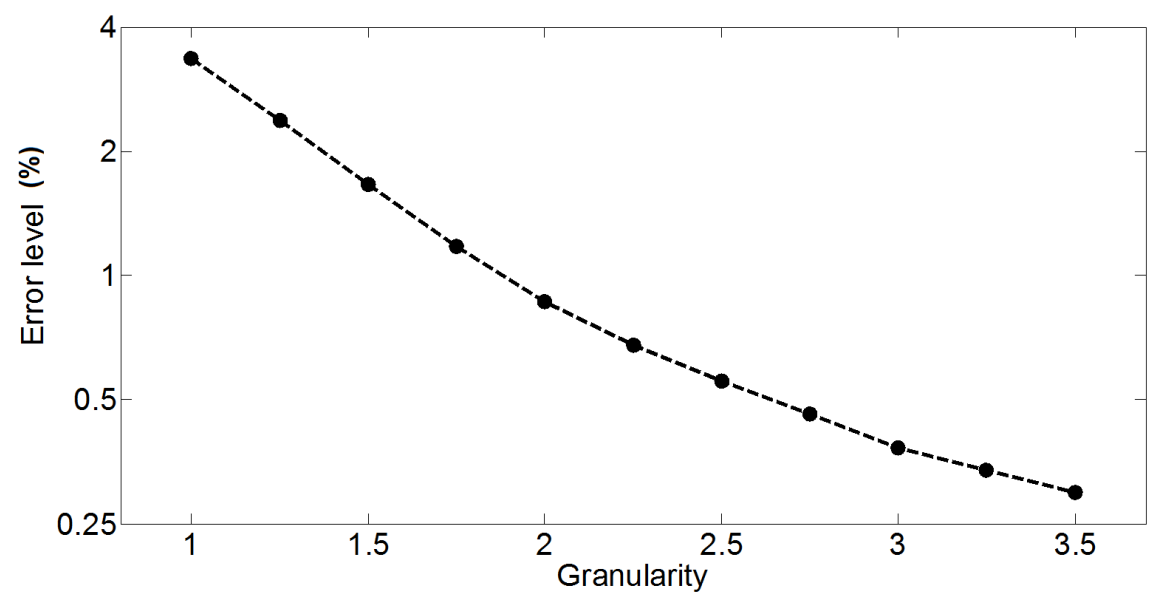

Figure 5

The geometric estimation error as a function of the applied resolution (granularity represent the applied average division factor)
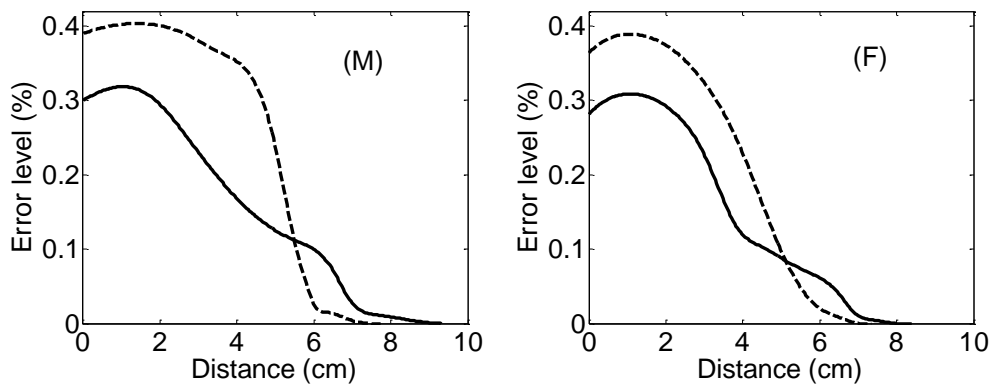

Figure 6

The geometric estimation error for a male $(\mathrm{M})$ and a female $(\mathrm{F})$ heart. The solid line represents the left, while the dashed line shows the right ventricle estimation error as a function of distance from the AVnode (base of ventricles)

Figure 7 exhibits the tissue orientation modeling: the image (A) visualizes the tissue fibers orientation in a sagittal segment, while images (B-D) shows the intersection of the ventricles using a horizontal plane. In all cases the applied planar resolution was $0.5 \mathrm{~mm}$, while the modeled slices (images (B)-(D)) were situated at $4 \mathrm{~cm}$ distance. 
Table I presents four important medical parameters (maximal and minimal interior volume, ejection fraction and stroke volume) of the modeled left ventricle. The simulation was performed for male and female hearts.

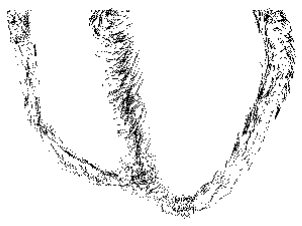

(A)

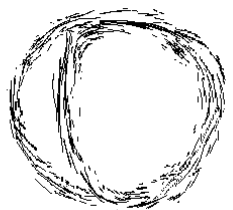

(C)

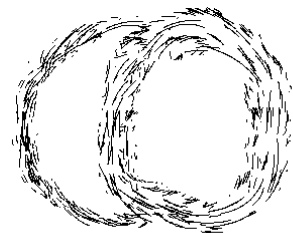

(B)

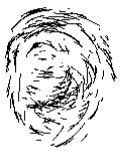

(D)

Figure 7

The simulated fiber orientations in the ventricular tissue. Image (A) visualizes the tissue fibers orientation in a sagittal segment, while images (B-D) show consecutive intersection of the ventricles from $\mathrm{AV}$-node to apex using a horizontal plane

Table I

Left ventricle parameters in function of gender

\begin{tabular}{|l|c|c|c|}
\hline & Male & Female & Average \\
\hline Max. volume (ml) & $105 \pm 31$ & $87 \pm 25$ & $96 \pm 28$ \\
\hline Min. volume (ml) & $48 \pm 15$ & $42 \pm 13$ & $45 \pm 14$ \\
\hline Ejection percentage & $57 \pm 15$ & $54 \pm 12$ & $55 \pm 13$ \\
\hline Stroke volume (ml) & $61 \pm 23$ & $49 \pm 21$ & $55 \pm 22$ \\
\hline
\end{tabular}

\section{Discussion and Conclusion}

An efficient ventricle geometry estimation method has to solve the continuous and flat transform among the basis voxels. As presented in Section 2.3, the aimed resolution can be acquired using fractional magnification factor. In this case the selected triangles have to be divided in such a way that the resulting new ones are as close to equilateral form as possible. This task can be solved by dividing the longer side-edges.

The increased curvature values demands a higher resolution for the starting base object, otherwise the resulting object may contain much higher estimation errors (see Figure 5). In this study all measurement tests were done with optimal conditions, so an improper division of the triangles may produce higher estimation errors than the measured ones. 
The multi-layer structure of the ventricular muscle was considered as a fuzzy-like system, where the different layers cannot be separated clearly, as the different cardiac muscle cell types may mix among themselves. In this mixed representation all generated compartments contain a unique cell type. The probability of a compartment to become a small cluster of a certain cell type is determined by the place of compartment and the ventricles geometry. Once its cell type is determined, it remains constant during the whole simulation. The movement of compartments was performed by maintaining the elementary connections among adjacent elements, maintaining the law of minimal internal tension.

In the case of healthy adult subjects, the shape of ventricles becomes less complicated, at the apex region, thus the level of estimation error decreases progressively with the distance from the AV-node (see Figure 6). In the case of infant heart modeling or in presence of several pathologies, these estimation errors become higher. It is also observable that in the case of male heart the organ size and estimation uncertainty is significantly higher. The increased incertitude level of male tissue estimation is due its higher mass, large size and less uniform shape. This form may produce higher pumping power, but at the price of higher risk of heart attack or formation of arrhythmias.

In agreement to the fact that the right ventricle is shorter than the left one, the estimation error for the right ventricle becomes zero at $7 \mathrm{~cm}$ or higher distance. The estimation error level of the right ventricle is significantly higher than the estimation error of the left ventricle, as the shape of the right ventricle may vary more intensely due to its thinner structure and significantly lower internal blood pressure. The estimation of apex region can be performed much efficiently because its shape is determined by the cardiac muscle and the level of internal blood has a relatively lower impact on it.

The fiber orientation of the ventricles may be determined more precisely than atria fiber direction (see Figure 7). Anatomically the ventricles possess a higher importance than atria and the genetic selection pressure induces a much more standardized ventricular muscle. The fiber direction of the left ventricle has an approximately circular structure because that form may produce an almost optimal mechanical contraction and pumping efficiency.

It was considered important to provide a resolution independent description of the heart, to make connections between medical parameters and graphical representations. The presented method may produce a much more compact representation of the ventricles and can enhance the performance of heart modeling and visualization algorithms.

\section{Acknowledgements}

This paper is a result of the project "Transnational Network for Integrated Management of Postdoctoral Research in Communicating Sciences. Institutional building (postdoctoral school) and fellowships program (CommScie)" - 
POSDRU/89/1.5/S/63663, financed under the Sectoral Operational Program Human Resources Development 2007-2013.

\section{References}

[1] Hill, J. A., Olson, E. N.: Muscle: Fundamental Biology and Mechanisms of Disease, Academic Press, London, Elsevier Inc. 2012

[2] Winfree, A. T.: Electrical Turbulence in Three-Dimensional Heart Muscle, Science 266 (1994) 1003-1006

[3] Panfilov, A. V.: Three-Dimensional Organization of Electrical Turbulence in the Heart, Physical Review E 59 (1999) R6251-R6254

[4] Sainte-Marie, J., Chapelle, D., Cimerman, R., Sorine, M.: Modeling and Estimation of the Cardiac Electromechanical Activity, Computers \& Structures 84 (28) (2006) 1743-1759

[5] Coghlan, H. C., Coghlan, A. R., Buckberg, G. D., Cox, J. L.: The Electrical Spiral of the Heart: its Role in the Helical Continuum. The Hypothesis of the Anisotropic Conducting Matrix, European Journal of Cardio-Thoracic Surgery 29 (1) (2006) S178-S187

[6] Caillerie, D., Mourad, A., Raoult, A.: Toward a Fiber-based Constitutive Law for the Myocardium, in: Proceedings of Modeling and Simulation for Computer-Aided Medicine and Surgery (2002) 25-30

[7] Antzelevitch, C., Shimizu, W., Yan, G. X., Sicouri, S., Weissenburger, J., Nesterenko, V. V.: The M Cell: Its Contribution to the ECG and to Normal and Abnormal Electrical Function of the Heart, Journal of Cardiovascular Electrophysiology 10 (9) (1999) 1124-1152

[8] Wong, J., Kuhl, E.: Generating Fibre Orientation Maps in Human Heart Models using Poisson Interpolation, Computer Methods in Biomechanics and Biomedical Engineering, (2012), DOI:10.1080/10255842.2012.739167

[9] Kumar, V., Abbas, A. K., Fausto, N.: Robbins and Cotran Pathologic Basis of Disease. Philadelphia: Elsevier Saunders. 2005

[10] Opie, L. H.: Heart Physiology: from Cell to Circulation. Philadelphia: Lippincott Williams \& Wilkins. 2003

[11] Sosnovik, D. E., Wang, R., Dai, G., Wang, T., Aikawa, E., Novikov, M., Rosenzweig, A., Gilbert, R. J., Wedeen, V. J.: Diffusion Spectrum MRI Tractography Reveals the Presence of a Complex Network of Residual Myofibers in Infarcted Myocardium. Circ: Cardiovasc Imaging. 2(3) (2009) 206-212

[12] Schmitt, B., Fedarava, K., Falkenberg, J., Rothaus, K., Bodhey, N. K., Reischauer, C., Kozerke, S., Schnackenburg, B., Westermann, D., Lunkenheimer, P. P. et al.: Three-Dimensional Alignment of the Aggregated Myocytes in the Normal and Hypertrophic Murine Heart. J Appl Physiol. 107 (2009) 921-927 
[13] Libby, P., Bonow, R. O., Mann, D. L., Zipes, D. P.: Braunwald's Heart Disease. Philadelphia: Saunders. 2007

[14] Streeter, D. D., Hanna, W. T.: Engineering Mechanics for Successive States in Canine Left Ventricular Myocardium: II Fiber Angle and Sarcomere length. Circ Res. 33(6) (1973) 656-664

[15] Arts, T., Costa, K. D., Covell, J. W., McCulloch, A. D.: Relating Myocardial Laminar Architecture to Shear Strain and Muscle Fiber Orientation. Am J Physiol Heart Circ Physiol. 280(5) (2001) H2222-H2229

[16] Geerts, L., Bovendeerd, P., Nicolay, K., Arts, T.: Characterization of the Normal Cardiac Myofiber Field in Goat Measured with MR-Diffusion Tensor Imaging. Am J Physiol Heart Circ Physiol. 283(1) (2002) H139H145

[17] Göktepe, S., Kuhl, E.: Electromechanics of the Heart - a Unified Approach to the Strongly Coupled Excitation Contraction Problem. Comput. Mech. 45 (2010) 227-243

[18] Takayama, K., Ashihara, T., Ijiri, T., Igarashi, T., Haraguchi, R., Nakazawa, K.: A Sketch-based Interface for Modeling Myocardial Fiber Orientation that Considers the Layered Structure of the Ventricles. J Physiol Sci. 58(7) (2008) 487-492

[19] Toussaint, N., Stoeck, C. T., Sermesant, M., Kozerke, S., Batchelor, P. G.: Three-Dimensional Prolate Spheroidal Extrapolation for Sparse DTI of the in Vivo Heart. In: Book of Abstracts of the International Society in Magnetic Resonance in Medicine. Sweden: Stockholm. 2010

[20] Kotikanyadanam, M., Göktepe, S., Kuhl, E.: Computational Modeling of Electrocardiograms - a Finite Element Approach towards Cardiac Excitation. Int J Numerical Methods Biomed Eng. 26 (2010) 524-533

[21] Nielsen, P. M., Le Grice, I. J., Smaill, B. H., Hunter PJ. 1991. Mathematical Model of Geometry and Fibrous Structure of the Heart. Am J Physiol Heart Circ Physiol. 260(4) H1365-H1378

[22] Zolgharnein, E., Mirsalimov, V. M.: Nucleation of a Crack under Inner Compression of Cylindrical Bodies. Acta Polytechnica Hungarica 9(2) (2012) 169-183

[23] Taheri, R., Mazaheri, K.: Hydrodynamic Optimization of Marine Propeller Using Gradient and Non-Gradient-based Algorithms. Acta Polytechnica Hungarica 10(3) (2013) 221-237

[24] Szilágyi, S. M., Szilágyi, L., Benyó, Z.: Volumetric Analysis of the Heart using Echocardiography. Lecture Notes in Computer Science 4466:81-90 (2007), ISSN: 0302-9743

[25] Delaunay, B.: Sur la sphère vide, Izvestia Akademii Nauk SSSR, Otdelenie Matematicheskikh i Estestvennykh Nauk, 7 (1934) 793-800 